\title{
NEUROENDOCRINE MARKERS AND SUSTENTACULAR CELL COUNT IN BENIGN AND MALIGNANT PHEOCHROMOCYTOMAS - A COMPARATIVE STUDY
}

\author{
Magdalena Bialas ${ }^{1}$, Krzysztof OKoń ${ }^{1}$, Grzegorz Dyduch ${ }^{1}$, \\ Katarzyna Ciesielska-Milian ${ }^{1}$, Monika Buziak ${ }^{2}$, Alicja Hubalewska-DydejczyK ${ }^{2}$, \\ MANUEL SOBRINHO-SimOES 3,4
}

\author{
${ }^{1}$ Chair of Pathomorphology, Jagiellonian University Medical College, Krakow, Poland \\ ${ }^{2}$ Chair of Endocrinology, Jagiellonian University Medical College, Krakow, Poland \\ 3Institute of Molecular Pathology and Immunology of the University of Porto (IPATIMUP), Porto, Portugal, \\ ${ }^{4}$ Medical Faculty, Department of Pathology, University of Porto, Porto, Portugal
}

\begin{abstract}
Pheochromocytomas are rare tumours with uncertain clinical behaviour. Histological separation between benign and malignant pheochromocytomas is usually difficult. The utilization of PASS criteria (Pheochromocytoma of the Adrenal Gland Scaled Score) has not provided a solid basis for separating benign from malignant tumours. The aim of this study was to investigate immunohistochemical markers (chromogranin, synaptophysin, S-100 and $\mathrm{Ki}-67$ ) to find out if they could provide useful diagnostic and/or prognostic data in a series of 62 pheochromocytomas ( 5 cases followed an aggressive clinical course). Chromogranin and synaptophysin immunoreactivity proved to be diagnostically useful, allowing, together with the absence of immunoreactivity for inhibin and melan $A$, an unequivocal diagnosis of pheochromocytoma. The pattern of staining did not provide, however, significant prognostic information. The mean count of sustentacular S-100 positive cells was lower in malignant than in benign pheochromocytomas but the frequent architectural variability and the haemorrhagic and cystic changes make it very difficult to achieve a precise and reproducible count in the majority of tumours. Without questioning that the occurrence of metastases and/or recurrent disease still remains the only unquestionable criterion for diagnosing a malignant pheochromocytoma, we think that the combined use of the PASS score and Ki-67 index provides useful information for diagnosing malignancy.
\end{abstract}

Key words: benign pheochromocytoma, metastasising pheochromocytoma, diagnosis, immunohistochemistry.

\section{Introduction}

Pheochromocytomas are rare, catecholamine-producing neuroendocrine tumours arising from chromaffin cells of the adrenal medulla $[1,2]$. Usually they are diagnosed in the fourth and fifth decade of life [3-5], but they can occur at any age - the cases of early onset are most commonly associated with hereditary syndromes: multiple endocrine neoplasia type 2 syndrome (MEN 2), neurofibromatosis (NF) or von Hippel-Lindau syndrome (VHL) $[6,7]$. The majority of pheochromocytomas are detected because of symptoms associ- 
ated with hormone production and secretion: sustained or paroxysmal hypertension, sweating, palpitations or headaches [8]. About $25 \%$ of tumours are non-secreting and are found incidentally during imaging studies of the abdomen performed for unrelated reasons (so-called incidentalomas) [8]. Malignant pheochromocytomas are rare - they represent about 5 to $13 \%$ of all cases $[2,6,9-11]$. The incidence of malignancy in tumours that arise on the background of a germline $S D H B$ mutation is higher, up to $30 \%$ [7, 12]. The histological separation between benign and malignant pheochromocytomas is usually very difficult and the only unquestionable criterion for diagnosis of malignancy still remains the presence of metastases (defined by the finding of tumour cells in sites where chromaffin cells are normally absent to distinguish metastases from multi-focal disease) or, according to some authors, also recurrent disease $[1,6]$. The most common metastatic sites are lymph nodes, liver, lungs, and bones [2]. $\mathrm{Pa}-$ tients with isolated bone metastases had better prognosis than patients with liver and lung metastases [13]. The 5-year survival rate for patients with malignant pheochromocytoma was $44-53 \%$ [14]. There is no single histological or immunohistochemical criterion which may predict the malignant behaviour of any single tumour, thus justifying the efforts that are being made to find a group of features which may allow one to separate benign from malignant tumours. The best known and most widely used multifactorial histological system is PASS (Pheochromocytoma of the Adrenal Gland Scaled Score) proposed by Thompson in 2002 [15]. This system is rather complicated and includes twelve histological features with different relative impacts. There is growing evidence that PASS is not a perfect tool to distinguish benign from malignant pheochromocytomas and that it has some limitations [16]. The inter-observer and intra-observer agreements have been reported to be unsatisfactory by some authors [17-19].

The aim of this study was to determine whether the use of several immunohistochemical markers could provide useful data for diagnosing and for predicting the clinical outcome of patients with pheochromocytoma in everyday pathomorphological practice.

\section{Material and methods}

A retrospective study of pheochromocytomas diagnosed in the Pathology Department of Jagiellonian University in Cracow during a period of 15 years (from 1996 to 2010) was performed. The records of $274 \mathrm{pa-}$ tients with symptomatic and asymptomatic adrenal tumours were reviewed in order to search for the diagnosis of pheochromocytoma. Sixty-seven tumours with this diagnosis were found in the files of the Pathology Department.

Haematoxylin and eosin-stained slides from 64 tumours were looked up (in three cases the slides were not available). All tumours were re-evaluated regarding their immunoreactivity for the following antigens (Table I). Briefly, a representative section from each tumour was selected for immunohistochemical investigation and $4 \mu \mathrm{m}$ sections were prepared. The immunohistochemistry was performed by the standard method: the slides were de-waxed, rehydrated and incubated in 3\% peroxide solution for 10 minutes to block the endogenous peroxidase activity. Antigen retrieval was carried out by microwaving in citrate buffer (for chromogranin, melan A and Ki-67) and EDTA (for synaptophysin and inhibin) for 5 minutes at $700 \mathrm{~W}$, then for 5 minutes at $600 \mathrm{~W}$. The Lab-Vision detection system was used. The primary antibodies and the respective technical details are summarised in Table I.

Chromogranin and synaptophysin staining was done to confirm the neuroendocrine nature of the tumours and S-100 to identify the presence of sustentacular cells. Additional reactions with melan A and inhibin antibodies were performed to evaluate the immunoreactivity of pheochromocytoma cells with these two markers, which are known to be diffusely present in lesions derived from the adrenal cortex. In each case, the proliferation activity was estimated as the percentage of tumour cells positive for the proliferation marker Ki-67.

Staining intensity with chromogranin and synaptophysin antibodies was graded as weak $(1+)$, moderate $(2+)$ or strong $(3+)$ and the percentage of positive cells was assessed. For Ki-67 antibody each slide was examined under low power to identify the areas

Table I. Primary antibodies used for immunohistochemical analysis

\begin{tabular}{lcccc}
\hline Primary antibody & Pre-Treatment & MANufacturer & $\begin{array}{c}\text { Dilution/ } \\
\text { Incubation Time }\end{array}$ & $\begin{array}{c}\text { Detection } \\
\text { MethoD }\end{array}$ \\
\hline chromogranin & citrate buffer & Dako & $1: 600 / 30 \mathrm{~min}$ & LabVision \\
\hline synaptophysin & EDTA & Dako & $1: 100 / 30 \mathrm{~min}$ & LabVision \\
\hline S-100 & - & Dako & $1: 400 / 30 \mathrm{~min}$ & LabVision \\
\hline melan-A & citrate buffer & LabVision & $1: 20 / 30 \mathrm{~min}$ & LabVision \\
\hline inhibin & EDTA & Dako & $1: 25 / 60 \mathrm{~min}$ & LabVision \\
\hline Ki-67 & citrate buffer & Dako & $1: 100 / 60 \mathrm{~min}$ & LabVision \\
\hline
\end{tabular}


with the highest density of Ki-67 positive cells. The proliferative index was expressed as a percentage of positively stained cells (counting at least 200 cells in $5 \mathrm{HPF}$ ). S-100 positive cells were counted in ten different, randomly selected high power fields (HPF).

Two out of the 64 selected cases were reclassified as cortical tumours - one as adrenocortical carcinoma (ACC) and another as adrenocortical adenoma (ACA) - and were excluded from the study. Finally we had analysed 62 pheochromocytomas diagnosed in 58 patients (four patients: three women and one man had bilateral tumours).

Five of the 58 patients with pheochromocytoma (8.6\%) had malignant tumours (three pheochromocytomas gave distant metastases and two recurred). All pheochromocytomas with malignant clinical course were unilateral. We obtained data on the follow-up of 48 of the other 57 cases (mean time of the follow-up: 45.6 months, median: 38 months). Nine cases were lost for follow-up, but we know they were not treated for any recurrence and/or metastasis in our department.

The Adrenal Gland Scaled Score (PASS) was evaluated according to the recommendations [15] in every pheochromocytoma $(n=62)$ by one pathologist (MB) who was not aware of the clinical history of the cases.

\section{Results}

The HE slides and paraffin blocks of the 62 cases of pheochromocytoma were available for reviewing and recutting. Two of the cases represented composite pheochromocytoma + ganglioneuroma. The group included 28 females (three had bilateral tumours) and 30 males (one had bilateral tumours) ranging in age from 19 to 75 years (mean age 47.6 years, median: 48 years, SD: 15.4). The mean age of male patients was higher than in the female group (48.4 years, range 19-75, $\mathrm{SD}=15.5$ and 46.9 years, range $19-73, \mathrm{SD}=15.5$ respectively). Thirty-four tumours $(54.8 \%)$ were found in the right adrenal whereas the left adrenal was involved in 24 cases (in four cases the site of the tumour was unknown) (Table 2). Seven patients were known to have one of the syndromes associated with increased incidence of adrenal pheochromocytoma (two patients with MEN 2A syndrome, four with NF and one with
Table II. Characteristics of patients with the diagnosis of pheochromocytoma

\begin{tabular}{lccc}
\hline & Male & Female & Total \\
\hline Age $(\mathrm{y})$ & 48.4 & 46.9 & 47.6 \\
\hline Site & & & \\
\hline left & 11 & 17 & 28 \\
\hline right & 17 & 13 & 30 \\
\hline unknown & 3 & 1 & 4 \\
\hline Diameter $(\mathrm{cm})$ & 5.1 & 4.9 & 5.0 \\
\hline PASS & 4.09 & 4.25 & 4.17 \\
\hline
\end{tabular}

VHL syndrome). Three of these patients (two with MEN 2A and one with NF syndrome) had bilateral tumours. The mean tumour diameter was $4.98 \mathrm{~cm}$ (median: $4.2 \mathrm{~cm}$, range $1.5-13 \mathrm{~cm}, \mathrm{SD}=2.38$ ) with no significant difference between male and female groups (5.1 and $4.9 \mathrm{~cm}$ respectively). Five tumours displayed cysts filled with blood with only a thin rim of neoplastic tissue at the periphery - the mean tumour diameter in this group was higher $(6.9 \mathrm{~cm})$ than in the entire pheochromocytoma group. Five out of 62 analysed tumours behaved in a malignant way (two of them recurred and three gave rise to distant metastases) - the mean tumour diameter in this group was $8.10 \mathrm{~cm}$ (median $7.0 \mathrm{~cm}$, range: $5.5-13 \mathrm{~cm}, \mathrm{SD}=2.88$ ) (Table III).

The mean PASS score in the entire analysed group was 4.36 with no difference between male and female patients ( 4.35 and 4.37 , respectively). In 28 cases ( $45 \%$ of the tumours) the PASS score was lower than 4 and in 34 cases ( $55 \%$ of the tumours) it reached or exceeded the limit of 4 points. In the latter group, 16 tumours scored 4 or 5 and in 18 tumours PASS reached 6 or more points. In the group of five tumours which displayed malignancy the mean PASS score was 8.6 (median 9.0, range $6-11 \mathrm{~cm}, \mathrm{SD}=2.51$ ) (Table III).

A positive reaction with synaptophysin was observed in all 62 pheochromocytomas. The reaction was present in almost all tumour cells (more than 90\%) (Fig. 1). This reaction was described as granular cytoplasmic staining with minimal variation of intensity: in 41 tumours $(66.2 \%)$ the intensity was described

Table III. Statistical comparison of size, PASS score and Ki-67 index in malignant (5 cases) and remaining non-malignant 57 cases

\begin{tabular}{|c|c|c|c|c|c|c|c|c|c|c|c|c|c|}
\hline \multirow[t]{2}{*}{ MALIGNANT } & \multicolumn{4}{|c|}{ DIAM } & \multicolumn{4}{|c|}{ KI67 } & \multicolumn{5}{|c|}{ PASS } \\
\hline & MEAN & $\mathrm{SD}$ & MIN & MAX & MEAN & SD & MIN & MAX & MEAN & SD & MIN & MAX & MEDIAN \\
\hline no & 4.70 & 2.16 & 1.50 & 11.00 & $1 \%$ & 0.005 & $0 \%$ & $4 \%$ & 3.93 & 2.05 & 0.00 & 9.00 & 4.00 \\
\hline yes & 8.10 & 2.88 & 5.50 & 13.00 & $3 \%$ & 0.022 & $1 \%$ & $6 \%$ & 8.60 & 2.51 & 6.00 & 11.00 & 9.00 \\
\hline \multirow[t]{2}{*}{ all cases } & 4.98 & 2.38 & 1.50 & 13.00 & $1 \%$ & 0.009 & $0 \%$ & $6 \%$ & 4.36 & 2.43 & 0.00 & 11.00 & 4.00 \\
\hline & \multicolumn{4}{|c|}{$\mathrm{U}=43.0, \mathrm{p}<0.0073$} & \multicolumn{4}{|c|}{$\mathrm{U}=62.0, \mathrm{p}<0.036$} & \multicolumn{5}{|c|}{$\mathrm{U}=20.5, \mathrm{p}<0.0004$} \\
\hline
\end{tabular}




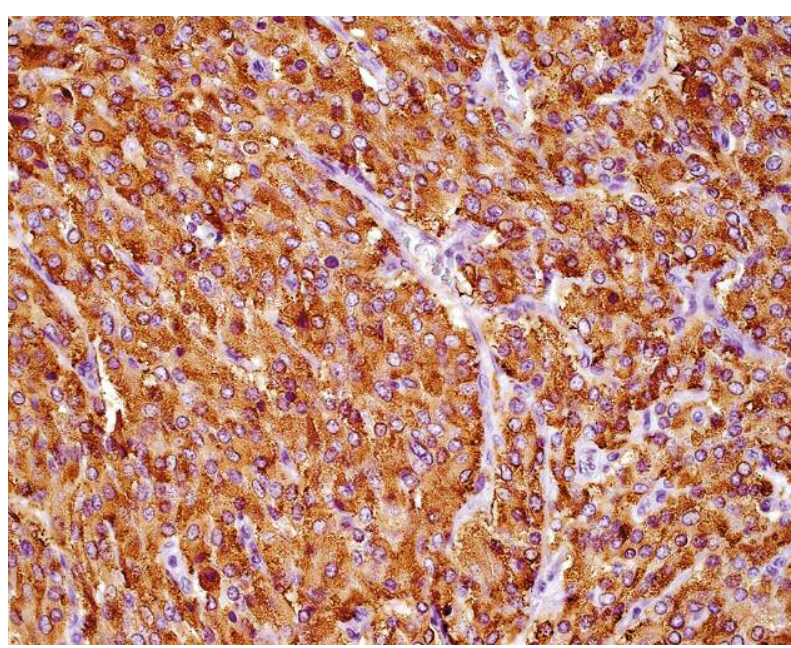

Fig. 1. Immunohistochemistry for synaptophysin: granular cytoplasmic staining with homogeneous moderate intensity is visible in the majority of cells

as medium $(2+)$ and in 21 tumours $(33.8 \%)$ as strong $(3+)$. Each of 62 tumours with diffuse synaptophysin immunoreactivity also displayed a diffuse and strong reaction with chromogranin in almost every cell (more than 90\%). The chromogranin staining was described as granular and cytoplasmic - staining intensity varied in the different areas of each tumour, as well as in different compartments of each cell, were observed (Fig. 2). The intensity of reaction was described as strong $(3+)$ in 34 cases $(54.8 \%)$, both strong and medium $(3+/ 2+)$ in 24 cases $(38.7 \%)$, and medium $(2+)$ in 4 cases $(6.5 \%)$. None of the 62 tumours with strong and diffuse synaptophysin and chromogranin immunoreactivity expressed melan A or inhibin.

The two tumours that were excluded from this series had small groups of cells presenting weak $(1+)$ expression for synaptophysin; one of them was negative for chromogranin and in the other weak expression of chromogranin was present in some cells. Both tumours

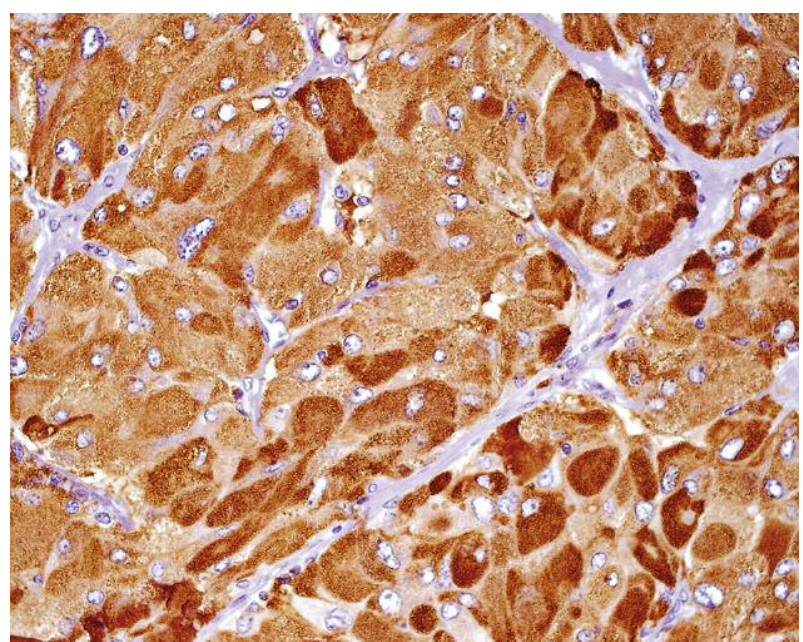

Fig. 2. Immunohistochemistry for chromogranin: granular cytoplasmic staining is present in the majority of cells. Variation in staining intensity is well visible

had strong and diffuse immunoreactivity with melan A. They also presented weak expression of inhibin in some cells. S-100 positive elongated cells were not present. Consequently, these two tumours were re-classified as adrenal cortical neoplasms and were excluded from the study.

S-100 antibody staining revealed the presence of S-100 positive elongated cells in all 62 pheochromocytomas. S-100 positive cells were present at the periphery of so-called cell nests in the areas where the nests were preserved. In the areas with solid cell growth or in tumours which presented as cystic neoplasms filled with blood, the distribution of S-100 positive cells was very uneven. There was a difference between mean count of S-100 positive cells in benign and malignant pheochromocytomas (Table 4) but the precise counting of sustentacular cells was very difficult; the S-100 positive cell count was different in different parts of the same tumour (Fig. 3A,B). Consequently the consistency of the results was unsatisfactory.
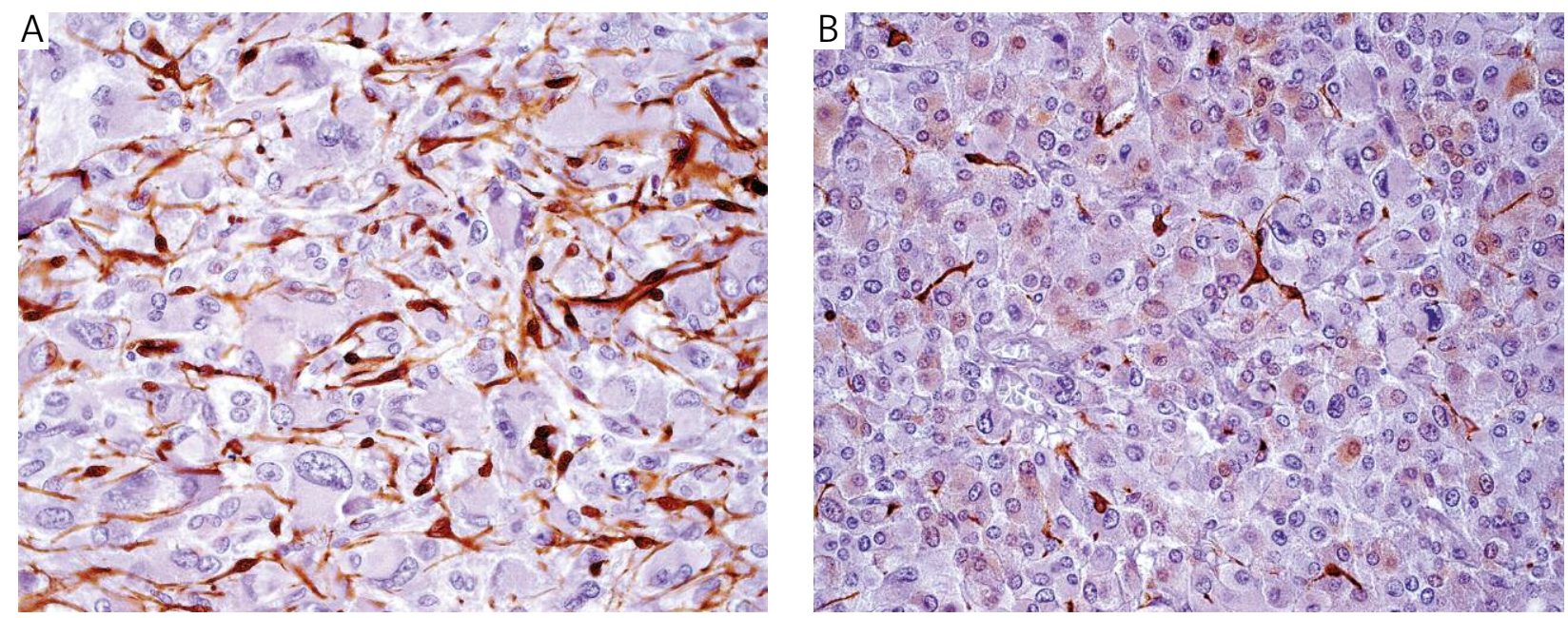

Fig. 3. In most tumours density of S-100 positive sustentacular cells was not uniform: within an individual (single) tumour, areas with high (A) and low (B) density were observed 
Table IV. Statistical comparison of mean count of S-100 positive sustentacular cells in benign and malignant pheochromocytomas

\begin{tabular}{lccc}
\hline Pheochromocytoma & $\begin{array}{c}\text { Mean } \\
\text { COUNT }\end{array}$ & Median & SD \\
\hline benign & 27.5 & 27 & 17.9 \\
\hline malignant & 22.2 & 19 & 8.43 \\
\hline
\end{tabular}

Regarding proliferation rate, 59 out of 62 tumours had a Ki-67 index between 0 and 2\% (Table V). In three tumours the proliferation rate was higher than $2 \%$. Two of them turned out to be malignant in the clinical course: the first was metastatic and had a proliferation activity of $6 \%$ (Fig. 4); the second recurred and had a proliferation rate of $4 \%$. The other pheochromocytoma with a proliferation rate of $4 \%$ was a $1.5 \mathrm{~cm}$ tumour found in the patient with MEN 2A syndrome who had bilateral pheochromocytomas but no signs of recurrence or metastasis 48 months after the operation.

\section{Discussion}

In 64 tumours diagnosed originally as pheochromocytoma, immunohistochemical analysis with a panel of six antibodies was performed. Both chromogranin and synaptophysin immunoreactivity was diffusely present in the 62 cases whose diagnosis was confirmed. The remaining two cases were reclassified as cortical tumours. The 62 pheochromocytomas did not express melan A or inhibin. Immunoreactivity for chromogranin and synaptophysin was described as diffuse, granular cytoplasmic staining with variation in the intensity of reaction in different areas of the same tumour, and for chromogranin, also in single cells. This variation was more pronounced for chromogranin and the overall staining intensity was stronger for chromogranin than for synaptophysin. There was no difference in the intensity of staining between small and large tumours, nor between those with benign and malignant clinical outcome. Immunohistochemistry is crucial to distinguish cortical from medullary adrenal tumours, but it is not helpful in predicting clinical behaviour of a pheochromocytoma.

A typical pheochromocytoma has an "organoid" structure which means that neoplastic cells are arranged in small, usually roundish nests (referred to as "Zellballen") separated by thin vascular septa and surrounded by elongated Schwann-like S-100 positive sustentacular cells $[1,6]$. In real life, however, the presence of well-defined, regular cell nests throughout the entire tumour is rarely observed. Usually, irregularly shaped, larger nests of neoplastic cells and trabecular areas, as well as more solid areas, are seen at least in some parts of the tumours. The organoid architecture of the neoplasm is even more disorganised in tumours
Table V. Proliferation rate in pheochromocytomas with benign and malignant clinical course

\begin{tabular}{lcc}
\hline Clinical course & \# OF CASES & $\begin{array}{c}\text { Proliferation } \\
\text { RATE }\end{array}$ \\
\hline $\begin{array}{l}\text { benign (no metastases } \\
\text { no recurrent) }\end{array}$ & 56 & less than 2\% \\
\hline $\begin{array}{l}\text { malignant } \\
\text { (metastases) }\end{array}$ & 1 & $4 \%$ \\
\hline $\begin{array}{l}\text { malignant } \\
\text { (recurrent) }\end{array}$ & 1 & less than 2\% \\
\hline
\end{tabular}

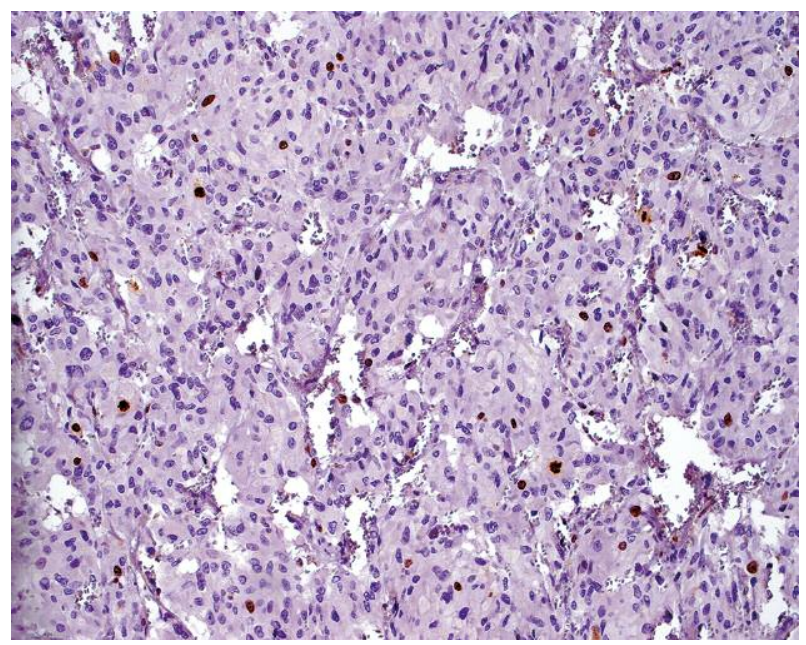

Fig. 4. Malignant pheochromocytoma: immunohistochemistry for Ki-67

with areas of haemorrhage, myxoid or hyaline stromal change and stromal fibrosis. The reaction for S-100 protein revealed the presence of sustentacular cells in all the 62 pheochromocytomas and absence of such cells in the two cases reclassified as cortical tumours. Sustentacular cells were present at the periphery of cell nests in the areas where the nests were preserved, and were more chaotically distributed in the areas with solid growth. The mean count of the S-100 positive sustentacular cells in high power fields was very uneven and the score was dependent on the shape and size of the cell nests. In the areas where cell nests were irregular and bigger, S-100 positive cells were less numerous but it was easier to count them. On the other hand, in the areas where cell nests were roundish and small, sustentacular cells were more numerous and had longer, branching projections, making the precise counting very difficult. We found significant differences in sustentacular cell number in different areas of the same tumour. We also found a difference in mean count of S100 positive cells between benign pheochromocytomas and the metastasizing or recurrent ones, but the final number of sustentacular cells in any tumour depended on the areas chosen for counting. The consistency 
of the results was unsatisfactory. Some authors found an association between lower average count of S-100 positive sustentacular cells in intra-adrenal pheochromocytomas or extra-adrenal tumours (paraganglioma) and unfavourable prognosis $\{20,21]$. Others stated that tumours with complete absence of sustentacular cells in some areas had greater potential for metastasis [22]. In our opinion, the sustentacular cell count is not reproducible in the majority of pheochromocytomas because of great variability of architectural patterns in most tumours, as well as because the frequent extensive haemorrhagic and cystic changes significantly disturb the network of S-100 positive cells. That is why the number of sustentacular cells cannot be used as a tool in everyday pathomorphological practice and is not helpful in predicting the clinical behaviour of pheochromocytomas.

The proliferation rate was estimated as the percentage of tumour cells positive with the proliferation marker $\mathrm{Ki}-67$. As $\mathrm{Ki}-67$ protein is present in nuclei during the G1, S, G2 and M phases of the cell cycle (but not in G0 phase), Ki-67 nuclear expression is characteristic of cells in the cell cycle [23]. The proliferation activity of tumour cells is a useful marker for estimating the growth potential in many tumours. Some authors have found a positive correlation between Ki-67 score (proliferation activity of the tumour) and malignant behaviour in pheochromocytomas [9, 24, 25, 26]. However, the Ki-67 score is not a perfect tool: the score in most pheochromocytomas is low, between 0 and $2 \%$ $[10,22,26]$. In the present study, not only 56 uneventful pheochromocytomas but also three which had a malignant clinical course had a proliferation activity between 0 and $2 \%$. These results correspond with those recorded in the literature $[10,22,26]$. On the other hand, two malignant, metastatic tumours had a proliferation rate $4 \%$ or higher. So it is probable that tumours with high proliferation activity (over $4 \%$ ) will behave malignantly, but the proliferation activity lower than $4 \%$ does not exclude the malignant potential of the tumour. One tumour with proliferation activity of $4 \%$ (found in a MEN 2A patient who had bilateral pheochromocytomas) did not show evidence of malignancy during the 24-month follow-up period, but the follow-up time is probably too short to allow any definitive conclusion. Van der Harst et al. reported that no benign pheochromocytomas exhibited a proliferative index greater than $2.5 \%$, whereas $50 \%$ of malignant tumours in their series did [22].

In conclusion:

1. Chromogranin and synaptophysin are useful markers to confirm the neuroendocrine nature of the tumours and to make a diagnosis of pheochromocytoma, but the pattern of staining is not helpful in predicting the malignant potential of the tumour.

2. Great variability of architectural patterns in any particular tumour, often extensive haemorrhagic changes and occasional cystic changes disturb the network of sustentacular S-100 positive cells, thus making it impossible to achieve a precise and reproducible sustentacular cell count in the majority of tumours. That is why the mean sustentacular cell count is not helpful in predicting clinical outcome of pheochromocytoma.

3. High Ki-67 is quite specific but not sensitive enough for diagnosing or at least predicting malignant behaviour. There is strong evidence to follow up more carefully patients with pheochromocytomas with a $\mathrm{Ki}-67$ index of $4 \%$ or higher. We have also obtained evidence favouring the possibility of using Ki-67 together with PASS in the identification of cases that may constitute a group of tumours with malignant potential: every tumour with a PASS score of 6 or more and $\mathrm{Ki}-67$ index of $2 \%$ or more should be followed closely.

4. Because of the above listed limitations, the presence of metastases and/or recurrent disease still remain the only unquestionable criterion for diagnosis of malignant pheochromocytoma.

This work was supported in part by Polish Ministry of Education grant no. N402 684340 awarded to Magdalena Biatas.

The authors declare no conflict of interest.

\section{References}

1. Miettinen M. Paragangiomas. In: Modern Soft Tissue Pathology - Tumors and Non-neoplastic Conditions. Miettinen M (ed). Cambridge University Press, New York 2010; 755-774.

2. Lack EE. Tumors of the adrenal glands and extraadrenal paraganglia. AFIP Atlas of Tumor Pathology. ARP Press Silver Spring, Maryland 2007; 241-281.

3. McNicol AM. Update on tumours of adrenal cortex, pheochromocytoma and extra-adrenal paraganglioma. Histopathology 2011; 58: 155-168.

4. Bialas M, Okon K, Stachura J. Primary adrenal tumors - a 16year experience in a single institution. Pol J Pathol 2008; 59: 101-106.

5. Goldstein RE, O’Neill JA, Holcomb GW, et al. Clinical experience over 48 years with pheochromocytoma. Ann Surg 1999; 229: 755-766.

6. DeLellis RA, Lloyd RV, Heitz PU, et al. Tumours of Endocrine Organs - Pathology and Genetics, WHO Classification of Tumours. IARC Press, Lyon 2004; 147-158.

7. Jafri M, Maher ER. The genetics of phaeochromocytoma: using clinical features to guide genetic testing. Eur J Endocrinol 2012; 166: 151-158.

8. Lenders J, Eisenhefer G, Mannelli M, et al. Pheochromocytoma. Lancet 2005; 366: 665-675.

9. de Wailly P, Oragano L, Rade F, et al. Malignant pheochromocytoma: a new malignancy criteria. Langenbecks Arch Surg 2012; 397: 239-246.

10. Strong VE, Kennedy T, Al-Ahmadie H, et al. Prognostic indicators of malignancy in adrenal pheochromocytomas: clinical, histopathologic and cell cycle/apoptosis gene expression analysis. Surgery 2008; 143: 759-768.

11. Lloyd RV. Adrenal cortical tumors, pheochromocytoma and paragangioma. Mod Pathol 2011; 24 Suppl 2: 58-65. 
12. Maitra A. The endocrine system. In: Robbins and Cotran Pathologic Basis of Disease. Kumar V, Abbas A, Fausto N, Aster J (eds.). $8^{\text {th }}$ edn. Saunders Elsevier, Philadelphia 2010; 1097- 1164.

13. Pacak K, Eisenhofer G, Ahlman H, et al. Pheochromocytoma: recommendations for clinical practice from the First International Symposium. October 2005. Nat Clin Pract Endocrinol Metab 2007; 3: 92-102.

14. Eisenhofer G, Bornstein SR, Brouwers FM, et al. Malignant pheochromocytoma: current status and initiatives for future progress. Endocr Relat Cancer 2004; 11: 423-436.

15. Thompson LD. Pheochromocytoma of the Adrenal gland Scaled Score (PASS) to separate benign from malignant neoplasms: a clinicopathologic and immunophenotypic study of 100 cases. Am J Surg Pathol 2002; 26: 551-566.

16. Agarwal A, Mehrotra PK, Jain M, et al. Size of the Tumor and Pheochromocytoma of the Adrenal Gland Scaled Score (PASS): Can They Predict Malignancy? World J Surg 2010; 34: 3022-3028.

17. Kajor M, Ziaja J, Lange D, et al. Analysis of morphology of adrenal pheochromocytoma as regards their potential malignancy. Endokrynol Pol 2005; 56(6): 911-916.

18. Wu D, Tischler AS, Lloyd RV, et al. Observer variation in the application of the Pheochromocytoma of Adrenal Gland Scaled Score. Am J Surg Pathol 2009; 33: 599-608.

19. Carlsen E, Abdullah Z, Kazmi SM, et al. Pheochromocytama, PASS and Immunohistochemistry. Horm Metab Res 2009; 41: 715-719.

20. Unger P, Hoffman K, Pertsemlidis D, et al. S-100 protein-positive sustentacular cells in malignant and locally aggressive adrenal pheochomocytomas. Arch Pathol Lab Med 1991; 115: 484-487.

21. Montresor E, Iacono C, Nifosi F, et al. Retroperitoneal paragangliomas: role of immunohistochemistry in the diagnosis of malignancy and in assessment of prognosis. Eur J Surg 1994; 160: $547-552$.

22. van der Harst E, Bruining HA, Bonjer J, et al. Proliferative index in pheochrmocytomas: does it predict the occurrence of metastases? J Pathol 2000; 191: 175-180.

23. Gerdes J, Lemke H, Baisch H, et al. Cell cycle analysis of a cell proliferation-associated human nuclear antigen defined by the monoclonal antibody Ki-67. J Immunol 1984; 133: 1710-1715.

24. Ohji H, Sasagawa I, Iciyangi Y, et al. Tumour angiogenesis and $\mathrm{Ki}-67$ expression in pheochromocytoma. BJU Int 2001; 87: 381-385.

25. Clarke MR, Weyant RJ, Watson CG, et al. Prognostic markers in pheochromocytoma. Hum Pathol 1998; 28: 522-526.

26. Brown HM, Komorowski RA, Wilson SD, et al. Predicting metastasis of pheochromocytoma using DNA flow cytometry and immunohistochemical markers of cell proliferation. A positive correlation between MIB-I staining and malignant tumour behaviour. Cancer 1999; 86: 1583-1589.

\section{Address for correspondence}

Magdalena Białas MD

Department of Pathomorphology

ul. Grzegórzecka 16

31-351 Kraków

tel. +48124211564

e-mail:mbialas7@gmail.com 